\title{
O Transtorno de Déficit de Atenção e Hiperatividade (TDAH) e as práticas pedagógicas em sala de aula
}

\author{
Attention Deficit Hyperactivity Disorder (ADHD) and pedagogical practices in the \\ classroom \\ El Trastorno del Déficit de Atención y Hiperactividad (TDAH) y las practicas pedagógicas \\ en el salón de clase
}

Luciana Teles Moura ${ }^{1}$, Katiane Pedrosa Mirandola Silva ${ }^{2 *}$.

\begin{abstract}
RESUMO
Objetivo: Revisar na literatura científica as práticas pedagógicas que devem ser utilizadas pelos professores de alunos com diagnóstico de Transtorno de Déficit de Atenção e Hiperatividade (TDAH) em sala de aula. Resultados e Discussão: Como resultado desse estudo foi possível identificar as principais práticas pedagógicas que devem ser utilizadas pelos educadores com estudantes portadores do TDAH, visando amenizar as dificuldades enfrentadas por esses alunos no processo de ensino-aprendizagem. Considerações finais: Pode-se concluir que a inserção de nova práticas pedagógicas no processo de ensino dos alunos TDAHs são necessárias e muito importantes, pois as mesmas buscam amenizar os desafios que os discentes enfrentam em sala de aula devido a esse transtorno. Espera-se, a partir desse estudo, contribuir de forma efetiva para que os professores de alunos diagnosticados com o TDAH reflitam em suas práticas pedagógicas, tornando sua atuação mais adequada, vislumbrando transpor as dificuldades vivenciadas pelos alunos TDAHs.
\end{abstract}

Palavras-chave: TDAH, Ensino, Aprendizagem, Escola.

\begin{abstract}
Objective: Review, in scientific literature, the pedagogical practices that should be used by teachers of students with a diagnosis of Attention Deficit Hyperactivity Disorder (ADHD) in the classroom. Results and Discussion: As a result of this study, it was possible to identify the main pedagogical practices that should be used by educators with students with ADHD, in order to alleviate the difficulties faced by these students in the teaching-learning process. Final considerations: It is possible to conclude that the insertion of new pedagogical practices in the teaching process of ADHD students is necessary and very important, because they seek to alleviate the challenges that students face in the classroom due to this disorder. This study, is expected to contribute effectively so teachers of students diagnosed with ADHD can reflect their pedagogical practices to make their actions more adequate, aiming to overcome the difficulties experienced by ADHD students.
\end{abstract}

Keywords: ADHD, Teach, Learning, School.

\section{RESUMEN}

Objetivo; Revisar, en la literatura científica las practicas pedagógicas que deben ser utilizadas por los profesores de alumnos con diagnóstico de Trastorno de Déficit de Atención y Hiperactividad (TDAH) en el salón de clase. Resultados y Discusión: como resultado de estos estudios fue posible identificar las principales practicas pedagógicas que deben ser utilizadas por los educadores con estudiantes portadores de TDAH, visando amenizar las dificultad enfrentadas por estos alumnos en el proceso enseño-aprendizaje.

${ }^{1}$ Doutorado em Psicologia pela Universidade Federal do Espírito Santo, Brasil

${ }_{2}^{2}$ Mestrado em Gestão Social, Educação e Desenvolvimento Regional pela Faculdade Vale do Cricaré - ES, Brasil. *E-mail: katianepms@yahoo.com.br 
Consideraciones finales: Puede-se concluir que la inserción de nuevas prácticas pedagógicas del proceso de enseño de los alumnos TDAH son necesarias y muy importantes, pues las mismas buscan amenizar los desafíos que los dicientes enfrentan en el salón de clase debido a este trastorno. Espera-se, a partir de estos estudios, contribuir de forma efectiva par que los profesores de alumnos diagnosticados con TDAH reflitan en sus prácticas pedagogicas, tornado su actuación más adecuada, vislumbrando transpor las dificultad vivenciadas por los alumnos TDAHs.

Palabras claves: TDAH, Ensino, Aprendizajem, Escuela.

\section{INTRODUÇÃO}

O direito à educação é amparado pela Política Nacional de Educação (PNE), independente de gênero, etnia, idade ou classe social. É um grande desafio à garantia de educação para todos, pois a inclusão de pessoas com deficiência, transtornos globais do desenvolvimento e altas habilidades ou superdotação em escolas comuns, de ensino regular, ampara-se na Constituição Federal de 1988, que define no artigo 205: "a educação como direito de todos, dever do Estado e da família, com a colaboração da sociedade, visando ao pleno desenvolvimento da pessoa, seu preparo para o exercício da cidadania e sua qualificação para o trabalho", e também garante, no artigo 208, o direito ao "atendimento educacional especializado aos portadores de deficiência" (BRASIL, 1988).

Diante disso, a escola deve possibilitar reais oportunidades de igualdade no ensino para todos os alunos, usando a flexibilidade nas práticas de ensino, atentando-se ao estilo de cada aprendiz, além de permitir a busca de novas alternativas e adaptações que facilitarão no processo de ensino-aprendizagem, sobretudo considerando-se que a inclusão constitui um grande desafio para as escolas regulares, que estão sendo chamadas para levar em conta a diversidade e as características e necessidades dos alunos, aderindo um modelo nele centrado e não no conteúdo, com destaque na aprendizagem e não, apenas, no ensino (CARVALHO RE, 2000).

A escola se depara com uma diversidade de alunos com os mais variados tipos de dificuldades de aprendizagens, entre muitos alunos que apresentam dificuldades, se destacam os diagnosticados com Transtorno do Déficit de Atenção e Hiperatividade (TDAH), objeto dessa pesquisa, que segundo Rohde LA e Benczik EB (1999), é um problema de saúde mental que se evidencia por desatenção, agitação ou hiperatividade e impulsividade. Devido aos problemas causados por esse transtorno na vida do aluno, surge a problemática em questão: Quais as práticas pedagógicas que devem ser utilizadas pelos professores com alunos portadores de transtorno de déficit de atenção e hiperatividade?

Procurando responder a esse questionamento, objetiva-se descrever as principais práticas pedagógicas que devem ser utilizadas pelos educadores de alunos TDAHs no processo de ensino-aprendizagem em sala de aula. Para tal pesquisa foi realizado um estudo de cunho bibliográfico visando traçar essas práticas e soluções pedagógicas que os docentes de estudantes TDAHs devem utilizar para amenizar as dificuldades que esse transtorno traz ao aluno em sala de aula.

\section{RESULTADOS E DISCUSSÃO}

Segundo Barkley R (2002), o TDAH é um transtorno do desenvolvimento do autocontrole que consiste em problemas com os períodos de atenção, o controle do impulso e o nível de atividade. A criança com esse transtorno apresenta dificuldades de controlar as emoções e o próprio comportamento.

Alguns estudiosos e autores defendem que se trata de um transtorno que é perceptível e facilmente identificável por todos que estão em constante contato com o portador, pois esses sintomas são visíveis, podendo causar estranheza em qualquer meio social (ALBANO AMS et al. 2012). Mediante isso, o relacionamento social do aluno com TDAH pode ser afetado devido os problemas causados por esse transtorno. 


\section{Tipos de TDAH}

De acordo com o DSM-V, O TDAH tem três tipos de classificações em seu diagnóstico: a primeira é a combinada, em que se encontram critérios tanto de desatenção quanto de hiperatividade - impulsividade; a segunda é a predominantemente desatento; sendo a terceira o predominantemente hiperativo - impulsivo (APA, 2013). Todos esses tipos podem afetar a vida escolar do aluno com TDAH devido seus problemas predominantes.

As pesquisas demonstram que o TDAH pode se apresentar acompanhado de outros distúrbios, que são chamados de comorbidades. Segundo Souza I, Pinheiro, MAS (2003), comorbidade é o termo utilizado para denominar a ocorrência de dois ou mais transtornos em uma mesma pessoa. Quando o aluno possui outros transtornos além do TDAH, o desafio para a escola é maior e se torna mais complexo de ser analisado. Então, deve-se contar com a ajuda dos pais, de especialistas e da escola para que esse aluno possa progredir como os demais.

O TDAH também pode ter comorbidade com os transtornos de aprendizagem, o que dificulta 0 desempenho escolar e acarreta em sofrimentos incalculáveis. Segundo discorre Silva ABB (2009), destacamse os seguintes: Dislexia: é um transtorno na área da leitura que se manifesta pela facilidade em trocar letras com diferenças sutis de grafias. Podem, também, trocar sílabas de uma palavra, dificultando a compreensão no momento da leitura; Disgrafia: apresenta dificuldade motora na execução da escrita. As letras podem ficar ilegíveis pela falta de harmonia nos movimentos e Discalculia: é um problema neurológico que dificulta ao indivíduo realizar operações matemáticas. Pode-se confundir números e ter enormes dificuldades em memorizá-los. Esses transtornos estão relacionados a problemas neurológicos e necessitam de um olhar diferenciado por parte da escola, além de uma equipe multidisciplinar para acompanhar o aluno em sua rotina estudantil, auxiliando em suas dificuldades apresentadas no momento da aprendizagem.

\section{O TDAH no âmbito escolar}

De acordo com a descrição no portal da ABDA - Associação Brasileira do Déficit de Atenção (2018), o TDAH é reconhecido oficialmente por vários países e pela Organização Mundial da Saúde (OMS). Em alguns países, como nos Estados Unidos, portadores de TDAH são protegidos pela lei quanto a receberem tratamento diferenciado na escola. É importante que a escola se preocupe em ofertar um tratamento diferenciado para esses estudantes, pois devido a esse transtorno surgem muitas dificuldades na sala de aula que podem interferir nesse processo de assimilação do conhecimento.

Considerando que a legislação brasileira deve garantir a todo aluno o direito à uma educação de qualidade, deve-se ressaltar que os professores têm papel fundamental nesse processo e precisam propiciar para essas crianças uma aprendizagem significativa. Por isso, é importante conhecer um pouco de cada aprendiz e suas particularidades e, acima de tudo, respeitar as diferenças e limitações de todo indivíduo, enquanto ser único, conforme evidencia o texto da Declaração de Salamanca, que as escolas devem adaptar-se a todas as crianças, independentemente das suas condições físicas, sociais, linguísticas ou outras. Neste conceito devem englobar-se crianças com deficiência ou superdotadas, crianças de rua ou crianças que trabalham, crianças de populações imigradas ou nômades, crianças de minorias linguísticas, étnicas ou culturais e crianças de áreas ou grupos desfavorecidos ou marginais (UNESCO, 1994).

Sendo assim, destaca-se que o direito à educação se dá independente das condições físicas, sociais, linguísticas, entre outras. E todos, precisam obter uma educação de qualidade, respeitando suas limitações. Dessa forma, a escola deve adaptar-se a todos, inclusive aos alunos com TDAH, buscando estratégias de ensino que contribuem para o desenvolvimento integral de todos em sala de aula.

A escola precisa dar suporte para que os professores aperfeiçoem seus conhecimentos e habilidades (CARVALHO RE, 2000), com o intuito de contribuir satisfatoriamente no aprendizado dos alunos com transtorno de déficit de atenção e hiperatividade. Os esforços em coordenar o TDAH por meio de apoio aos professores nas práticas pedagógicas são fundamentais para que os alunos com esse transtorno se sintam inclusos em todo processo de ensino aprendizagem. 
Albano et al. (2012) afirmam que é essencial que o professor não receie em experimentar novas práticas pedagógicas ativa e realmente significativa. Com isso, nota-se que as novas práticas pedagógicas são alicerçadas de estratégias de ensino diferenciadas que incentivam o aprendizado do aluno TDAH de forma expressiva.

Diante de todo esse cenário, é possível observar que a escola exerce uma grande influência na qualidade do ensino de seus TDAHs e o professor poderá contribuir, significativamente, usando algumas estratégias diversificadas. Então, Silva ABB (2009) sugere algumas dicas que poderão contribuir no gerenciamento do TDAH: professores devem ter conhecimento sobre esse transtorno, jogo de cintura e flexibilidade para entender como funciona a cabeça desse estudante para tentar ajudá-lo em sala de aula. E, seguindo essa linha de pensamento, a autora acredita também que os professores precisam manter a disciplina em sala de aula e exigir que os limites sejam obedecidos por todos, incluindo o aluno TDAH. E, para isso, faz-se necessário deixar as regras bem claras e explícitas e ter sempre uma dose extra de paciência (SILVA ABB, 2009).

Por conseguinte, a articulista também adverte que ter conhecimento sobre TDAH é primordial para saber lidar com esse tipo de educando. Manter contato com outros profissionais da escola e com o (s) médico (s) e/ou psicólogos que cuidam dessas crianças e/ou adolescentes é também muito importante; utilizar o estímulo sempre que possível, fazer elogios e proporcionar atividades que diminuam a inquietação e dispersão por meio de recursos como notebooks, palm tops e outros, são estratégias que segundo Silva ABB (2009), contribuem para um ensino mais significativo para esses estudantes.

Entende-se então, que a utilização de novas tecnologias pode influenciar diretamente no aprendizado de todos os alunos, e é um recurso muito interessante que pode contribuir para uma aprendizagem intensa. $\mathrm{E}$ na visão de Albano AMS et al. (2012), a tecnologia poderá ser vista como uma possibilidade de diferenciação do trabalho docente, em que o professor, por meio desses elementos tecnológicos, contribui para a compreensão do conteúdo em diferentes contextos. Esse recurso deve ser aliado a ludicidade e explorado na escola, por meio de orientações pedagógicas, estando de acordo com os objetivos traçados de cada atividade a ser desenvolvida.

Os autores destacam, ainda, a importância da figura do educador perante os educandos TDAHs: o professor deve ter a percepção de que a dinâmica familiar influencia, decisivamente, no surgimento de uma série de transtornos nos filhos, cabendo à escola a função de acolher esses pais para que haja um engajamento entre a escola e a família; o professor enquanto uma das principais referências para a criança, tem condições de perceber quando seus alunos se encontram em estado de sofrimento e de agir "preventivamente", encaminhando-os para um serviço de avaliação e atendimento de crianças (ALBANO AMS et al., 2012).

Com isso, os estudiosos acreditam que o educador, por meio de observações, deve estar sempre atento para perceber sintomas e indícios de riscos nos alunos, pois são indícios de risco para o desenvolvimento psíquico da criança: elevação da agressividade, desengajamento nas atividades acadêmicas, dificuldades em focar sua atenção, isolamento, entre outros (ALBANO AMS et al., 2012). E, a partir desses dados, encaminhar para uma avaliação com especialistas mantendo sempre contato com os familiares.

Partindo desse pressuposto, vê-se que o contato estreito com a família é muito importante, pois além do tratamento médico e/ou psicológico é primordial para a criança TDAH conviver em um ambiente confortável. Para Dupaul GJ e Stoner G (2007), as crianças que apresentam tanto TDAH quanto outros déficits nas habilidades acadêmicas vão necessitar de tratamento em múltiplos contextos, implementados por vários profissionais.

Segundo Oliver L (2011) e Chamat LSJ (2008), as crianças com TDAH (Transtorno de Déficit de Atenção e Hiperatividade) e Distúrbio do Déficit de Atenção (DDA) devem receber acompanhamento com psicopedagogo e, em casos mais graves, há necessidade de tratamento medicamentoso. A parceria dos especialistas na superação das dificuldades causadas pelo transtorno na vida do TDAH contribui muito na aprendizagem desses alunos em sala de aula. 
Diante dessa perspectiva, o professor deve permitir-se utilizar do poder de influência que exerce sobre o estudante, para auxiliá-lo na superação de suas dificuldades no ambiente da sala de aula; deve sempre "olhar" para os seus alunos supondo e apostando que eles poderão realizar tarefas e adquirir conhecimentos que ainda não possuem no momento; a expressão de afetos é muito importante para os TDAHs e isso pode ser permitido por meio da expressão dos conteúdos de forma diferenciada e lúdica como: via desenhos ou brincadeiras (ALBANO AMS et al., 2012). Proporcionar momentos de expressão de afeto, contribui para que esses alunos se sintam mais seguros em suas atividades escolares.

Albano AMS et al. (2012) apud Acúrcio e Andrade (2003), ressaltam igualmente a importância do jogo como estratégia de desenvolvimento de raciocínio, da lógica, como forma de entendimento e fixação de conteúdo. Propiciar ao aluno TDAH atividades por meio de jogos e brincadeiras, o coloca a cumprir tarefas seriamente que prendem a atenção, ao cumprimento de desafios e regras, tão importantes para o seu desenvolvimento. No momento do jogo, esse aluno busca possibilidades de superar algumas dificuldades em busca de alcançar o objetivo traçado, e atividades lúdicas podem contribuir na aprendizagem, pois o prazer e o desejo de aprender devem caminhar juntos (ALBANO AMS et al., 2012). O professor deve inserir essas atividades lúdicas em suas práticas pedagógicas, pois ajuda a criança a estabelecer vínculos positivos e a compreender situações de limites.

Por conseguinte, entende-se que as estratégias utilizadas por meio do lúdico, além de serem atividades agradáveis poderão contribuir muito para alcançar as habilidades previstas nas atividades diárias, e também contribuir para que a desatenção e a inquietude sejam amenizadas.

Tratar o aluno TDAH como sujeito desde pequeno, e não como alguém incapaz auxiliando a buscar ajuda quando se sentir ameaçado. Apresentar o combinado, de forma clara, antes do início de uma atividade e sempre retorná-lo quando necessário, são estratégias relevantes na sala de aula, de acordo com o ponto de vista de Albano AMS et al. (2012)

Portanto, intervenções comportamentais baseadas em sala de aula, para o TDAH, devem incluir os sistemas de reforço por fichas combinados com custo da resposta, nos quais as contingências estão disponíveis na escola, em casa ou em ambos os cenários, a fim de se motivar a criança a prestar atenção às tarefas designadas e às regras da sala de aula (DUPAUL GJ e STONER G, 2007). As intervenções são fundamentais na vida do TDAH por meio de atividades com reforços que contribuirão para a melhoria da obediência às regras, a atenção e as tarefas designadas.

Por consequência, outro destaque importante com TDAHs é a formação da imagem corporal, pois crianças que apresentam o sintoma de hiperatividade precisam executar atividades que propiciem a representação de seus corpos, já que elas têm uma imagem distorcida do próprio corpo (ALBANO AMS et al., 2012).

Phelan TW (2005) traz algumas sugestões que também podem ser úteis ao educador quanto ao estímulo para um melhor desempenho escolar de alunos com TDAH. São elas: limpar a área de trabalho, fragmentar a tarefa em unidades pequenas e administráveis, dar orientações para uma tarefa e fazer checagens frequentes para ter certeza de que a tarefa está sendo realizada. Diante disso, o professor deve sempre ajudar a manter a limpeza da carteira e os materiais dos TDAHs organizados, contribuindo para um momento mais tranquilo na realização das tarefas desse aluno.

O articulista ressalta também que tentar administrar as tarefas, dar somente as orientações necessárias e checar continuamente para verificar se elas estão sendo realizadas de acordo com o solicitado é sempre muito válido para essas crianças. Para Phelan TW (2005), essas estratégias também podem e devem ser aplicadas no seio familiar.

Dupaul GJ e Stoner G (2007), destacam algumas questões que merecem atenção durante o planejamento de intervenções comportamentais com os TDAHs: Crianças com diagnóstico de TDAH geralmente carecem de feedback mais frequente e específico que seus colegas para fortalecer o desempenho, precisam constantemente de reforços para salientar a atenção deles na execução das atividades na sala de aula, considerando que costumam dispersar rápido. 
Logo, o feedback do professor precisa ser mais frequente do que para os demais alunos, até sobre o desempenho deles para evitar a perda da motivação ou até algum tipo de frustração mediante às dificuldades do dia a dia.

Diante desse cenário, Dupaul GJ e Stoner G (2007) advertem que de forma geral, a quantidade de tarefas na sala de aula deve ser reduzida para os alunos TDAHs, e a complexidade dessas atividades deve ser aumentada gradualmente. E ainda alertam para outro fator relevante, que se trata de casos em que uma tarefa que seja voltada para a mesma habilidade ou área conceitual, pode ser substituída com o intuito de evitar o tédio e a exacerbação potencial dos problemas de atenção.

A dupla pondera ainda que as recompensas ou reforços podem ser programadas sempre ao término de uma tarefa em uma área de menor preferência, sendo sempre variados conforme necessário, atividades preferidas (por exemplo, tempo para atividades de livre escolha, acesso ao computador) devem ser usadas como reforços, em vez de recompensas tangíveis sempre que possível (DUPAUL GJ e STONER G, 2007).

Buscar entender os transtornos funcionais que existem na vida dos alunos, inclusive os portadores de TDAH, não é tarefa fácil, mas necessária para que o professor possa contribuir de forma satisfatória na busca de novas práticas pedagógicas, que atendam às necessidades específicas de cada aluno no processo de ensino aprendizagem.

\section{CONSIDERAÇÕES FINAIS}

Após verificar as informações encontradas na pesquisa, conclui-se que as dificuldades apresentadas por alunos TDAHs no processo de ensino podem ser amenizadas caso a escola possibilite a esses discentes, metodologias e intervenções que visam valorizar as potencialidades e criatividade dos estudantes portadores de TDAH.

Portanto, os professores desenvolvem um papel muito importante no processo de aprendizagem dos alunos com transtorno de déficit de atenção e hiperatividade. E, em função disso, precisam buscar cada vez mais conhecimentos sobre esse transtorno, para que possam manejar melhor as próprias aulas quando se depararem com as dificuldades encontradas em classe com esses alunos.

Compreende-se que a escola deve buscar, sempre que possível, formar e manter uma parceria com os pais de alunos, principalmente aqueles que tem dificuldades de aprendizagem como os diagnosticados com TDAH, tendo em vista que ambos contribuem no desenvolvimento das potencialidades desses alunos e podem, juntos, superar os obstáculos do cotidiano escolar, buscando sempre o bem-estar desses alunos por meio de um ensino eficaz.

Espera-se, a partir desse estudo, contribuir de forma efetiva para que professores de alunos TDAHs reflitam em suas práticas pedagógicas, tornando a própria atuação mais adequada, vislumbrando, assim, a transposição de dificuldades vivenciadas pelos estudantes portadores de TDAH.

\section{REFERÊNCIAS}

1. ABDA, Associação Brasileira do Déficit de Atenção. Manual diagnóstico e estatístico dos transtornos mentaisV.https://tdah.org.br/?s=Manual+diagn\%C3\%B3stico+e+estat\%C3\%ADstico+dos+transtornos+mentais+V Acesso em 14: set. 2018.

2. ALBANO AMS, VICENZI E, et al. A criança com TDAH: metodologias e adaptações curriculares. Curitiba: Fael, 2012.

3. APA, American Psychiatric Association. Manual Diagnóstico e Estatístico de Transtornos Mentais: DSM - V. 1ํㅡㄹ Ed. Porto Alegre: Artmed, 2013.

4. BARKLEY R. A. Transtorno de Déficit de Atenção/hiperatividade. São Paulo: Artmed, 2002.

5. BRASIL, Constituição Federal do Brasil. Brasília: Senado, 1988.

6. CARVALHO, RE. Removendo Barreiras para a Aprendizagem. Educação Inclusiva. Porto Alegre: Artes Médicas, 2000. 
7. CHAMAT LSJ. Técnicas de intervenções psicopedagógicas: para dificuldades e problemas de aprendizagem. São Paulo: Vetor, 2008.

8. DUPAUL GJ, STONER G. TDAH nas Escolas. São Paulo: M. Books do Brasil, 2007.

9. OLIVER L. Distúrbios de aprendizagem e de comportamento: Como detectar, entender e tratar os problemas de aprendizagem. Um guia indispensável para pais, professores e profissionais da saúde. Rio de janeiro: Wak Editora, 2011.

10. PHELAN TW. TDA/TDAH: Transtorno de déficit de atenção e hiperatividade. São Paulo: M Books do Brasil Editora Ltda., 2005.

11. ROHDE LA, BENCZIK EBP. Transtorno de Déficit de Atenção/Hiperatividade. O que é? Como posso ajudar? Porto Alegre: Artes Médicas; 1999.

12. SILVA ABB. Mentes inquietas: TDAH; desatenção, hiperatividade e impulsividade. 4. Ed. Rio de Janeiro: Objetiva, 2009.

13. SOUZA I, PINHEIRO MAS. Co-morbidades. In: Rohde, Luiz Augusto, Mattos, Paulo e cols. Porto Alegre: Artmed AS, 2003

14. UNESCO, Declaração de Salamanca. Salamanca: Conferência Mundial de Educação Especial, 1994. 